\title{
Utility of SanIA Chatbot to Maintain Continuity of Care and Psychological Support During COVID-19 Pandemic
}

\author{
César Morcillo Serra*1,4, Daniel Tizon Galisteo ${ }^{2}$, Soraya Bajat Pacios ${ }^{3}$, Ana Aroca Tanarro ${ }^{1,4}$, \\ Sonia Gutiérrez Gabriel ${ }^{4}$, Domingo Marzal Martín ${ }^{4}$ and José Francisco Tomás Martínez ${ }^{4}$ \\ ${ }^{1}$ Digital Hospital, Sanitas Hospitals, Spain
}

${ }^{2}$ Information technology systems and artificial intelligence, Sanitas Hospitals, Spain

${ }^{3}$ Psicology Department, La Zarzuela University Hospital, Sanitas Hospitals, Spain

${ }^{4}$ Medical Direction, Sanitas Hospitals, Spain

*Corresponding author: César Morcillo Serra, Medical Direction, Digital Hospital, Sanitas Hospitals, Pg Manuel Girona 33.08034

Barcelona, Spain

\section{ARTICLE INFO}

Received: 幽 February 04, 2021

Published: 幽 February 15, 2021

Citation: César Morcillo Serra, Daniel Tizon Galisteo, Soraya Bajat Pacios, Ana Aroca Tanarro, Sonia Gutiérrez Gabriel, et al. Utility of SanIA Chatbot to Maintain Continuity of Care and Psychological Support During COVID-19 Pandemic. Biomed J Sci \& Tech Res 33(5)-2021. BJSTR. MS.ID.005474.

Keywords: Digital Health; Chat bot; Artificial Intelligence; COVID-19

\section{ABSTRACT}

Introduction: Digital health facilitates accessible, safe and more efficient care, through technologies such as bots and artificial intelligence. Undoubtedly, its implementation has been accelerated thanks to the COVID-19 pandemic, where they have demonstrated their effectiveness, by maintaining continuity of care and facilitating early interventions, such as psychological support, that has been of vital importance in a crisis situation, where mental health problems have increased considerably.

Objective and Methods: Prospective observational study to describe the utility of SanIA chatbot during COVID-19 pandemic, focusing the psychological tool.

Results: During 2020, we have experienced an exponential increase in the number of SanIA consultations, with 824,435 conversations attended. Within its multiple functions it has performed 60,467 confirmed appointments, 160,422 appointments canceled, 136,432 online check-ins and 62,826 documents sent. One of its main functionalities is as a psychological bot that has carried out 6,915 psychological evaluations. Of these it has diagnosed $28 \%$ of costumers with depressed mood, $13 \%$ irritable mood, and $10 \%$ anxiety or panic attack disorder, doing 5,292 online mindfulness sessions via bot and generating 1,507 appointments with a specialist (psychologist, psychiatrist or neurologist).

Conclusion: Chatbot SanIA has helped to fight the COVID-19 crisis, making information available and maintaining continuity of care by providing advice, including psychological assessment, to patients whenever they want it $24 / 7$.

\section{Introduction}

The irruption of technology in healthcare is an increasingly evident reality, driven by the rise of digital health, which provides innovative patient-centered care solutions like artificial intelligence (AI) and bots, promoting accessibility, quality, safety and efficiency in all areas of health [1]. These new digital technologies allow building a different relationship with the patient, focused on their needs, transparent, agile and continuous 24 hours a day, where the user enjoys much more information, participation and autonomy. They have the potential to simplify both administrative and healthcare processes, to improve the quality and reduce the cost of medical care. AI is intended to redesign health care, by improving workflow and allowing patients to process their own data to promote their health, through mobile devices and applications that create the digital channel, where healthcare can be delivered anywhere [2]. AI can help the insufficient number of doctors to treat the growing number of patients more efficiently, through digital diagnosis and monitoring of diseases, through the use of interactive virtual assistants, which can be implemented on mobile phones around the world and facilitate universal access to low-cost, predictive, personalized healthcare [3]. 
Chatbots or conversational bots (shortened form of robot) are a great application of AI, which is a program that simulates having a conversation with a human being. It is able to process messages in natural language, issued by the user through keyboard or audio systems and it generates responses sent again to the user. These automated voice conversation virtual assistants are useful for managing tasks with minimal human-machine interaction, or for health problem management, such as helping to improve weight loss [4] or helping depression [5] or to deal with COVID-19 crisis [6]. Bots can make information available at a scale well beyond telemedicine approaches due to automation and this can provide support for people who cannot afford care, who can't communicate well in English, or who prefer anonymity. These approaches can be developed to tie to institution-specific resources and that will enhance customer service by providing the best answer to patients whenever they want it, without having to wait and without variability based on who answers the phone [7]. They are accessible any time, allowing patients to obtain answers $24 / 7$ and to avoid wait times on hold before reaching a human. Bots have a higher handling capacity than any human. A single chat bot can simultaneously have conversations with thousands of people no matter what time of day. Regardless of the volume of calls, every question may be answered immediately. The objective of $\mathrm{AI}$ is not to replace health personnel but to expand their skills, since its greatest potential lies in the analysis of large amounts of data that will allow replacing part of the healthcare staff routine work [8].

\section{Materials and Methods}

This is a prospective observational study to describe the utility of Sanitas SanIA chatbot during COVID-19 pandemic, between January and December, 2020. Sanitas is a health insurance company with around 2 million costumers, 5 teaching hospitals and many outpatient health care facilities throughout Spain. SanIA has multiple functions (Figure 1) like bureaucratic processes, online access to medical documentation and reports, self-evaluation of COVID-19 disease, and a self-assessment system of the user's mental health, validated by specialists in psychiatry and psychology, based on AI, with the aim of providing anyone with access to an immediate and free digital health resource, helping them to identify a health problem, saving distances and social stigma, and thus improving their quality of life. Access to the assistant is anonymous and universal through any device with internet access. The AI system is trained to use the same question circuits that are used in face-to-face psychological consultation and assesses whether the user exceeds certain thresholds related to common psychological problems. If necessary, it recommends a referral to the specialist. If they do not need it and they have adequate emotional well-being, mindfulness sessions are offered [9]. For the analysis of the data, usual descriptive statistical techniques have been used.
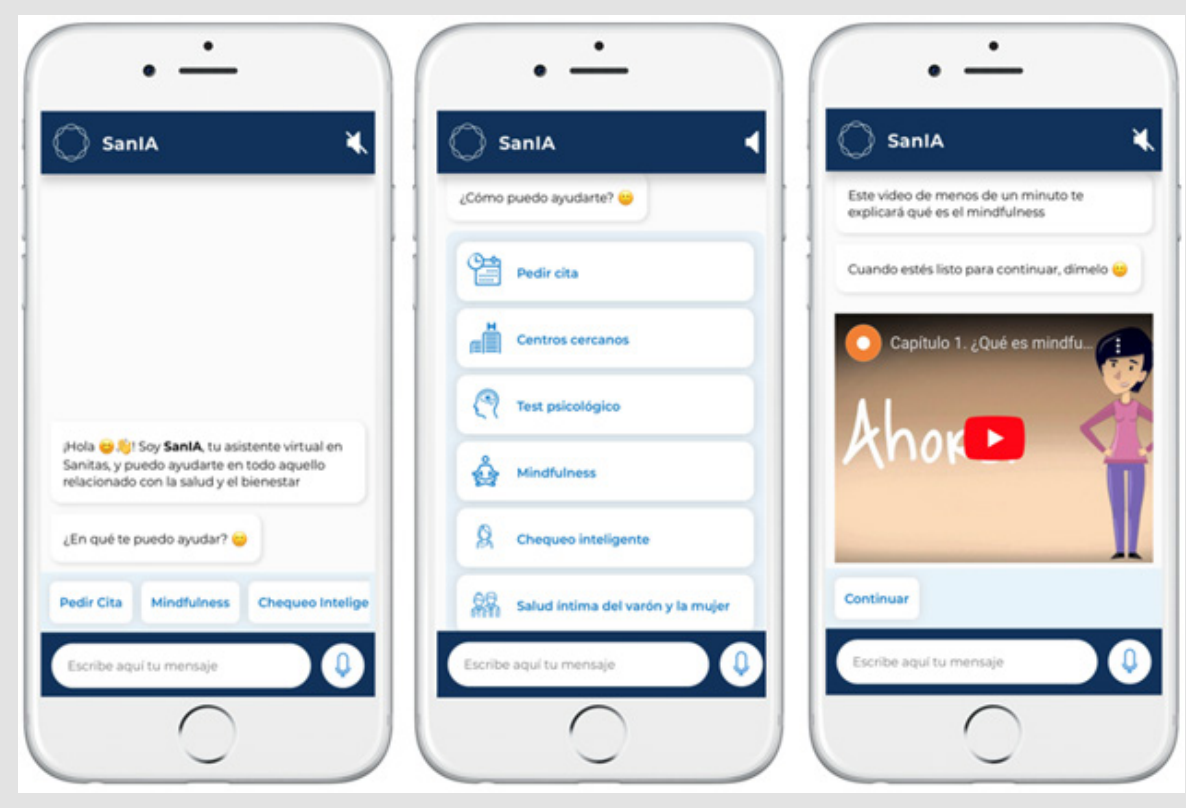

Figure 1: SanIA screenshots showing its multiple functions.

\section{Results}

During 2020, we have experienced an exponential increase in the number of SanIA consultations, with 824,435 conversations attended. SanIA has performed 60,467 confirmed appointments, 160,422 appointments canceled, 136,432 online check-ins and 62,826 documents sent. One of its main functionalities is as a psychological bot that has carried out 6,915 psychological evaluations (Figures $2 \& 3$ ). Of these it has diagnosed $28 \%$ of costumers with depressed mood, 13\% irritable mood, and 10\% anxiety or panic attack disorder among other problems. Figure 2 shows the prevalence of all detected psychological problems. SanIA has done 5,292 online mindfulness sessions (Figure 4) and 
generated 1,507 appointments with a specialist (psychologist, correlated with the incidence of COVID-19, with two peaks that psychiatrist or neurologist) (Figure 5). The activity of the bot is coincide with the first two waves of the disease in 2020 in Spain.

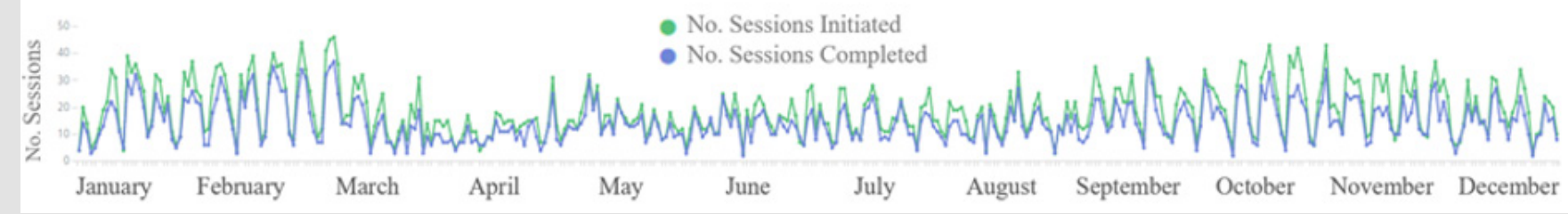

Figure 2: Number of SanIA psychological assessments during 2020.

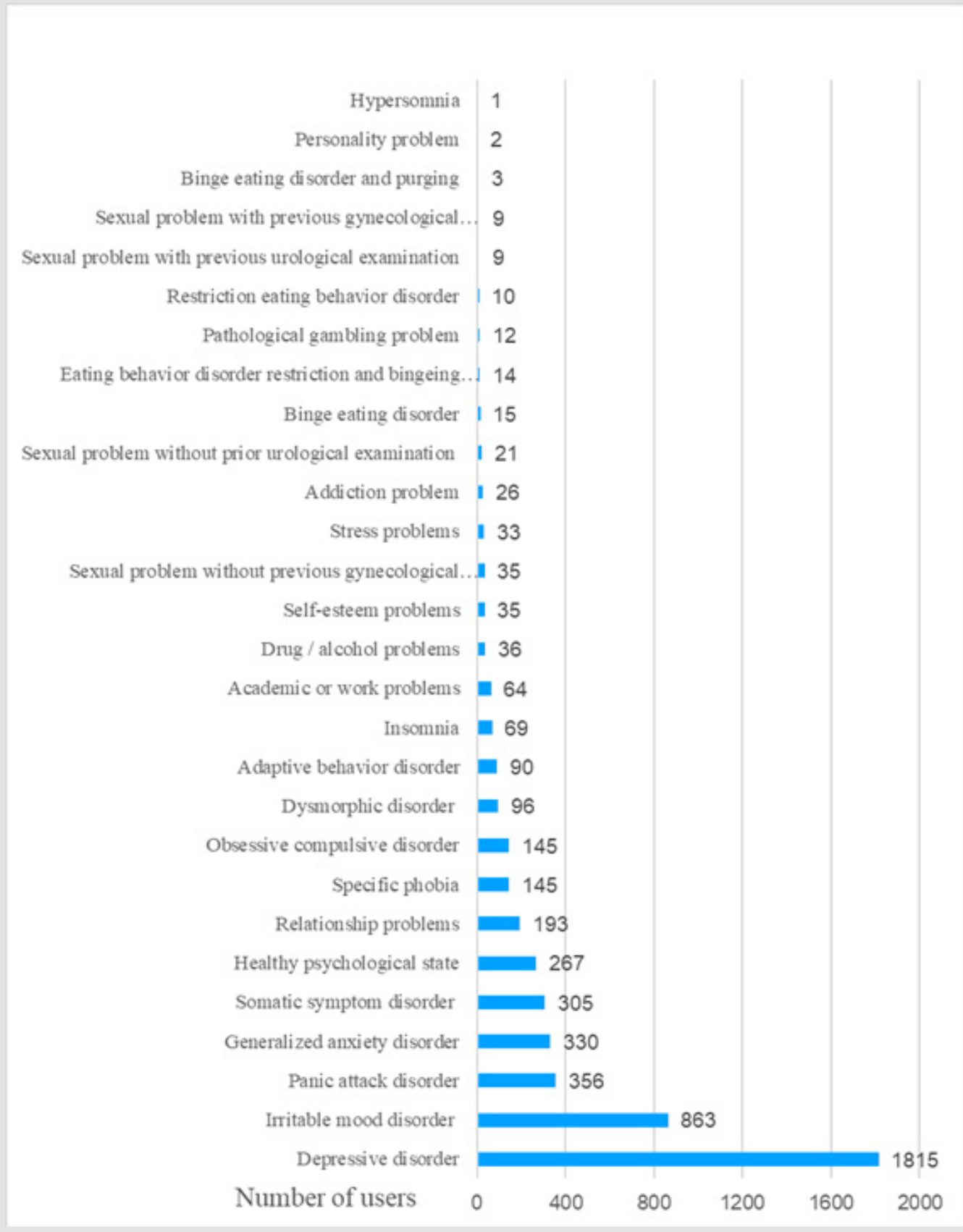

Figure 3: Prevalence of detected psychological problems. 


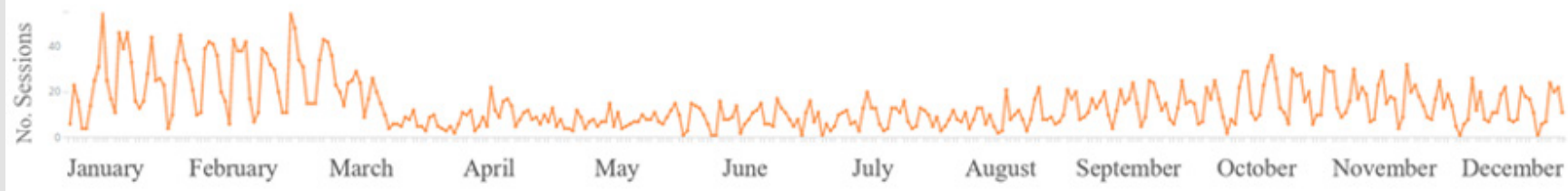

Figure 4: Number of SanIA mindfulness sessions during 2020.

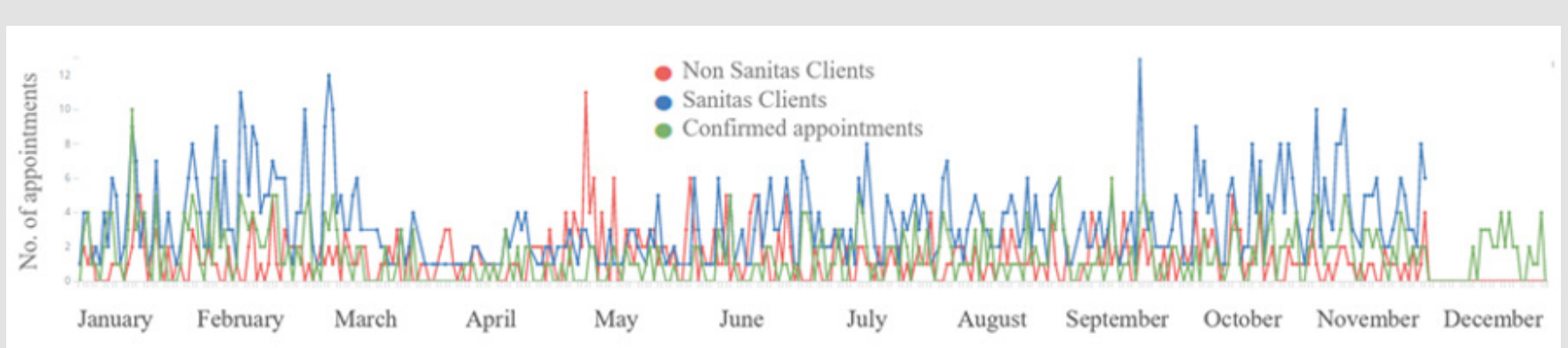

Figure 5: Number of appointments generated by SanIA with a specialist after psychological assessment during 2020.

\section{Discussion}

This study shows how the chatbot SanIA has helped to establish a formal and secure communication channel between the citizen and the health system, to maintain continuity of care by providing advice, including psychological assessment, to patients whenever they want it 24/7 [6]. This psychological support has been of vital importance in a crisis situation such as that generated by COVID-19, where mental health problems have increased considerably. This is reflected in the activity of the bot, that is correlated with the incidence of COVID-19, with two peaks that coincide with the first two waves of the disease in 2020 in Spain. Digital health tools have been shown to be the main driver for reducing bureaucratic processes related to health, which has saved time for patients and professional staff, avoided non-essential visits by citizens to health centers, and decreased risks of COVID-19 infection for both citizens and health professionals [10]. The COVID-19 pandemic has brought about a sudden change in the adoption of digital health strategies, which will undoubtedly continue in the long term. People respond positively to COVID-19 screening chatbots, but the primary factor driving user response to screening hotlines (human or chatbot) is perceptions of the agent's ability. When ability is the same, users view chatbots no differently or more positively than human agents. The primary factor driving perceptions of ability is the user's trust in the hotline provider, with a slight negative bias against chatbots' ability. So ensuring that COVID-19 screening chatbots provide highquality service is critical but not sufficient for widespread adoption. The key is to emphasize the chatbot's ability and assure users that it delivers the same quality as human agents [11]. For years, AI programmers became obsessed with passing the Turing test, the famous challenge launched by Alan Turing in 1950 to produce a machine that can trick a human into thinking they are also human.
Since the precursor of today's robots, ELIZA by Joseph Weizenbaum, created at M.I.T. In 1966, much progress has been made and the use of bots with AI to treat patients with psychological problems is already a reality [12]. In this way, the use of AI manages to break the taboo of verbally expressing your mood and begins to be aware of your illness [13]. Revealing personal information to another person has beneficial emotional, relational and psychological results.

When the interlocutor believes they are interacting with a computer rather than another person, such as a chatbot that can simulate a person-to-person conversation, emotional disclosures have been shown to be equivalent in conversations with a chatbot or a person [14]. Thanks to $\mathrm{AI}$, natural language processing, machine learning and virtual assistants, mental health problems can be addressed [15]. Chatbots can help users with depression or anxiety to restructure their situations using cognitive behavioral therapy techniques [16]. Woebot was created by leading experts in clinical psychology and has proven its ability to make people happier. Woebot is an automated conversation agent designed to deliver cognitive behavioral therapy in the form of short daily conversations and intelligent mood tracking. It is used within an instant messaging application that is platform independent and can be used on a computer or mobile device [17]. Wysa is an AI coach that helps you achieve your desired goals. The majority (79\%) of users improved their depression symptoms and $68 \%$ found the Wysa experience helpful and encouraging [18]. Tess is another mental health chatbot that trains people and offers evidence that AI can serve as an accessible and profitable therapeutic agent. Although not designed to take over the role of a trained therapist, integrative counseling emerges as a viable option for providing support [19]. Mental disorders are the leading cause of disability in the world and AI has been shown to improve the management 
of these health problems. The integration of AI, through chatbots and other tools such as facial recognition and voice analysis, allow diagnosing the presence of anxiety or depression from the mobile, alerting the user and the medical team of the patient's situation. These technologies can be deployed on mobile phones and facilitate low-cost universal access to pain and mental health care.

\section{Conclusion}

Chatbot SanIA has helped to fight the COVID-19 crisis, making information available and maintaining continuity of care by providing advice to patients whenever they want it $24 / 7$. The psychological virtual assistant tool has made it possible to assess a high proportion of mental health problems, to carry out mindfulness sessions and if necessary, to recommend a referral to the specialist. We have the opportunity to create new approaches based on AI to identify health problems and intervene early in clinically significant events, and thus improve the quality of life.

\section{References}

1. Morcillo C, González JL (2020) New digital healthcare technologies. Med Clin Barc 154(7): 257-259.

2. Rajkomar A, Dean J, Kohane I (2019) Machine Learning in Medicine. N Engl J Med 380(14): 1347-1358.

3. Topol EJ (2019) High-performance medicine: the convergence of human and artificial intelligence. Nature Medicine 25(1): 44-56.

4. Chang L, Booyoon C, Ga Hye Y, Bumjo O, Yun O (2018) Mobile health, physical activity, and obesity: Subanalysis of a randomized controlled trial. Medicine 97(38): e12309.

5. Barrett PM, Steinhubl SR, Muse ED, Topol EJ (2017) Digitising the mind Lancet 389(10082): 1877.

6. Morcillo C, Tizon D, Marzal D, Tomás JF (2020) Digital health solutions implemented by Sanitas hospitals to maintain continuity of care during COVID-19 pandemic. Medical Research Archives 8(8).

7. Herriman M, Meer E, Rosin R, Lee V, Washington V, et al. (2020) Asked and Answered: Building a Chatbot to Address Covid-19-Related Concerns. N Engl J Med Catalyst.

\section{ISSN: 2574-1241}

DOI: 10.26717/BJSTR.2021.33.005474

César Morcillo Serra. Biomed J Sci \& Tech Res

(C) This work is licensed under Creative

Submission Link: https://biomedres.us/submit-manuscript.php
8. Gruber K (2020) Is the future of medical diagnosis in computer algorithms? Lancet Digital Health 1(1): e15-e16.

9. Bajat Pacios S, Tizón Galisteo D, Ferri M, González Romero J, Morcillo Serra C (2020) Utilidad de un asistente virtual psicológico en la valoración del estado de ánimo. Rev Clin Esp 220: 729.

10. Hollander JE, Carr BG (2020) Virtually Perfect? Telemedicine for Covid-19. N Engl J Med 382: 1679-1681.

11. Dennis AR, Kim A, Rahimi M, Ayabakan S (2020) User reactions to COVID-19 screening chatbots from reputable providers. J Am Med Inform Assoc 27(11): 1727-1731.

12. He L, Cao C (2018) Automated depression analysis using convolutional neural networks from speech. J Biomed Inform 83: 103-111.

13. Barrett PM, Steinhubl SR, Muse ED, Topol EJ (2017) Digitising the mind. Lancet 389(10082): 1877.

14. Ho A, Hancock J, Miner AS (2018) Psychological, Relational, and Emotional Effects of Self-Disclosure After Conversations with a Chatbot. Journal of Communication 68(4): 712-733.

15. Triantafyllidis AK, Tsanas A (2019) Applications of Machine Learning in Real-Life Digital Health Interventions: Review of the Literature. J Med Internet Res 21(4): e12286.

16. Mohr DC, Riper H, Schueller SM (2018) A solution-focused research approach to achieve an implementable revolution in digital mental health. JAMA Psychiatry 75(2): 113-114.

17. Fitzpatrick KK, Darcy A, Vierhile M (2017) Delivering Cognitive Behavior Therapy to Young Adults with Symptoms of Depression and Anxiety Using a Fully Automated Conversational Agent (Woebot): A Randomized Controlled Trial. JMIR Ment Health 4(2): e19.

18. Inkster B, Sarda S, Subramanian V (2018) An Empathy-Driven, Conversational Artificial Intelligence Agent (Wysa) for Digital Mental Well-Being: Real-World Data Evaluation Mixed-Methods Study. JMIR Mhealth Uhealth 6(11): e12106.

19. Fulmer R, Joerin A, Gentile B, Lakerink L, Rauws M (2018) Using Psychological Artificial Intelligence (Tess) to Relieve Symptoms of Depression and Anxiety: A Randomized Controlled Trial. JMIR Ment Health 5(4): e64.

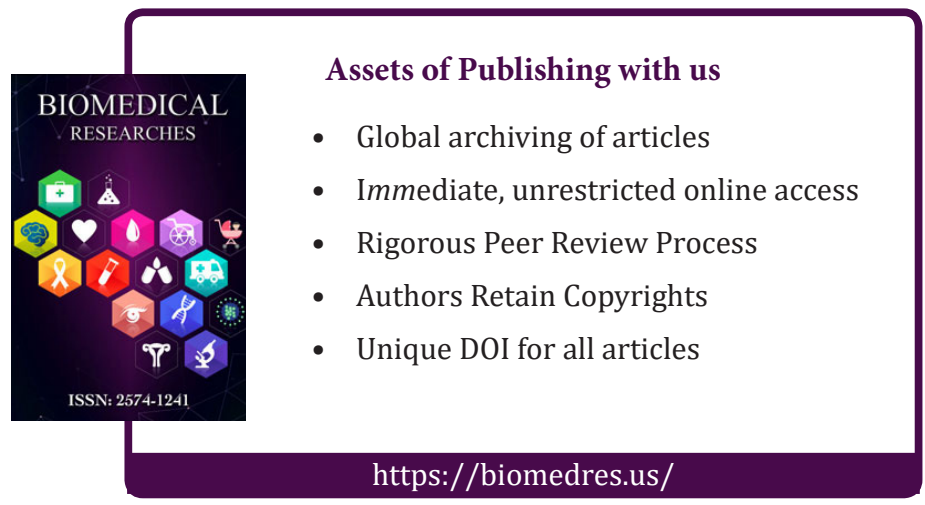

